\title{
La perspectiva del interaccionismo estructural para el análisis de redes sociales
}

Ainhoa de Federico de la Rúa -Département de sociologie et d'anthropologie, Université de Toulouse 2- Le Mirail CNRS-LISST-CERS

\section{Resumen}

El presente texto detalla el modelo teórico de actor de la aproximación llamada "interaccionismo estructural" para el análisis de redes sociales, así como sus implicaciones de cara al método de obtención de datos y de análisis de los mismos.

Se distinguen los dos grandes métodos: sociocéntrico y egocéntrico, derivados de la tradición histórica del estudio de las redes sociales (Moreno y Barnes). A modo de ilustración, se aplica dicha aproximación a los modelos de comprensión de la difusión y prevención del sida.

Palabras clave: interaccionismo estructural, modelo de actor, teoría, redes sociales, SIDA.

\begin{abstract}
This paper presents the theoretical model of the "structural interactionism" approach for the analysis of social networks. The implications of this theoretical approach on methods of data gathering and data analysis are explored. Two general methods derived from the historical tradition of the study of social networks (Moreno and Barnes) are distinguished: sociocentric and egocentric methods. To illustrate the "structural interactionist" approach and its implications, the model is applied to the comprehension of the diffusion of AIDS and its prevention.
\end{abstract}

Key words: structural interactionism, model of action, theory, social networks, AIDS.

Podemos encontrar el concepto de red en casi todas las disciplinas científicas, entre ellas las ciencias sociales, pero también se trata de un término que ha entrado en el vocabulario común en que tiene significados diversos. Cualquiera que sea la moda actual sobre dicha palabra, hace más de medio siglo que el concepto de red comenzó a adquirir una definición específica y a ser utilizado de forma precisa en ciencias sociales.

Podemos recordar a dos de los pioneros para mostrar cómo ambos lograron realizar una contribución al conocimiento a partir de marcos teóricos diferentes.

A principios de los años 30 en Estados Unidos, Jacob Moreno, psicólogo social de origen rumano, sugirió considerar como "átomo social” ( social atom »), no tanto al individuo, sino al individuo y las relaciones interpersonales de atracción y rechazo que se organizan a su alrededor. Esta visión reconoce en el campo intelectual de la psicología social un objeto intermediario entre el grupo, forma 
REDES- Revista hispana para el análisis de redes sociales

Vol.17,\#12, Diciembre 2009

http://revista-redes. rediris.es

elemental del orden social, y el individuo, receptáculo de las pasiones y de la personalidad. Al estudiar una institución de rehabilitación, Jacob Moreno se preguntó porqué algunas jóvenes se escapaban en serie y descubrió que estaban ligadas entre ellas y que formaban "una red" ( «a network»). En este concepto identificó la existencia de relaciones que iban más allá de la frontera de los grupos y les dio una posición teórica precisa. Primero, se trata de un fenómeno "estructurado": dichas redes de relaciones, tan informales como puedan parecer, no dejan de estar reguladas, organizadas, estructuradas, por principios que es preciso esclarecer. Segundo, Jacob Moreno atribuyó a dichas redes una función: permiten una cierta forma de comunicación de la información y contribuyen a la circulación de los rumores, y por lo tanto, a la formación de la opinión publica que asegura una función de regulación social. Son supra-individuales, pero están desligados de las organizaciones, que son capaces de trascender, son informales pero no informes y son funcionales. Así pues, en 1934, las redes adquieren sus títulos de nobleza teórica en el campo de la psicología social. 
REDES- Revista hispana para el análisis de redes sociales

Vol.17,\#12, Diciembre 2009

http://revista-redes. rediris.es

Veinte años más tarde, en el campo de la antropología social inglesa, disciplina también habituada a la observación directa de pequeños sistemas de interacción social, se descubre la importancia de ciertos sistemas de relaciones interpersonales. John Barnes estudia una parroquia Noruega. Le interesan dos cuestiones: el tipo de estratificación social y el funcionamiento del poder. En ella, distingue un campo de actividad económica ligado a la pesca, un campo territorial (vecindad y cooperación agrícola) y, por fin, un tercer campo que une los dos anteriores. Este último no comporta «ni unidades, ni fronteras ; ni organización de coordinación. Está constituido por los lazos de amistad y conocidos que todo nativo de Bremnes hereda en parte, pero que sobretodo instaura por si mismo [...] la mayor parte de dichos lazos existen entre personas que reconocen tener un estatus aproximadamente igual y, yo pienso, que se podría decir que estos lazos constituyen el sistema de clases de Bremnes ${ }^{1}$. » (Barnes, 1954, p. 237). Así pues Barnes puede retomar sus cuestiones. Por una parte, los actores evalúan su posición social dentro de la red en que están sobretodo en contacto con personas poco diferentes; por lo tanto, aunque saben que existen diferencias de riqueza, se pueden considerar como iguales. Por otra parte, si cada habitante está asociado a muchos otros en la red de formas diferentes, permitir que aparezcan oposiciones graves y conflictos pondría en peligro dichos lazos. La red tiene aquí como efecto funcional el evitar conflictos en las diferentes instancias de poder de la colectividad.

Ambos ejemplos son significativos de la variedad de aproximaciones que, al principio y a continuación, precisarán el concepto de red. Una de ella parte de las relaciones entre los individuos, los pequeños grupos, y desemboca en la idea de regulación social por las redes, crisol de la opinión pública. La otra parte de agrupaciones institucionales para reconstituir la matriz de las instituciones de una sociedad. Ambas reconocen por el camino las redes como una realidad estructurada y estructuradora que cumple funciones específicas. Ambas orientaciones, fundamentalmente contrastadas, siguen siendo hoy (más allá de los grandes desarrollos que se han producido en la disciplina ${ }^{2}$ ) una característica importante del vasto campo intelectual que se reconoce bajo la etiqueta de las redes.

\footnotetext{
${ }^{1}$ « The third social field has no units or boundaries; it has no coordinating organization. It is made up of the ties of friendship and acquaintance which everyone growing up in Bremnes societly partly inherits and largely builds up for himself. [...] Most of the ties are however, between persons who accord approximately equal status to one another, and it is these ties which, I think, may be said to constitute the class system of Bremnes. »

${ }^{2}$ Aquellos interesados en el desarrollo histórico general del análisis de redes pueden referirse a las obras de John Scott (1991) y Linton Freeman (2004).
} 
REDES- Revista hispana para el análisis de redes sociales

Vol.17,\#12, Diciembre 2009

http://revista-redes. rediris.es

\section{Redes observadas y sistemas relacionales}

\section{A) Dos métodos}

Como los pioneros antes citados, vamos a partir de consideraciones empíricas para definir el objeto de este artículo y diremos que una red de relaciones sociales es identificable si definimos un conjunto de actores, un tipo particular de relaciones, y que podemos observar las relaciones de ese tipo existentes o inexistentes entre esos actores. Si podemos examinar las relaciones entre cada actor y todos los demás, entonces hablaremos de red completa, global, total o sociocéntrica. Así pues podríamos observar mediante una encuesta las relaciones entre los miembros de una asociación, los concejales de un municipio, los profesionales de un servicio... que formen redes no demasiado grandes. Por otra parte, las relaciones objeto de registros sistemáticos permiten trabajar sobre redes completas de gran tamaño (a partir de datos administrativos, boletines, registros, etc.).

Si el conjunto de actores no está delimitado, se trata de una red abierta: una red de amistad, de parentesco, a menudo de vecindad, una red sexual... Por definición esa red no puede ser observada de forma exhaustiva. Entonces se procede mediante sondeo atomístico y se pide a cada encuestado que describa las personas con las que mantiene el tipo de relación examinada. Se llama estrella relacional al conjunto formado por las relaciones directas de un actor focal encuestado (que se llama ego) con otras personas (llamadas alter). Se hablará de red personal o egocéntrica si podemos pedir a ego que describa las relaciones entre los alteri (en inglés: personal community, ego network, personal network).

Se pueden situar dichas definiciones con referencia a objetos clásicos: los sistemas de parentesco son redes típicamente constituidas por tres tipos de lazos elementales: la alizanza, la consanguinidad y la filiación. La psicología social de los pequeños grupos se interesa también por las estructuras relacionales en los grupos y ciertos de sus efectos (comunicación, influencia) y se apoya a menudo en observaciones de laboratorio que permiten constituir pequeñas redes completas, pero que no tienen en cuenta las pertenencias simultáneas de los actores a diversos sistemas relacionales. Finalmente un análisis de redes es mucho más preciso que la mayoría de los estudios sobre la sociabilidad en la medida en que se recogen las propiedades específicas de cada relación con cada persona y su organización sociométrica global. Por ejemplo, la encuesta describe cada relación con los vecinos de ego y no lo que éste hace en general con los vecinos. 
REDES- Revista hispana para el análisis de redes sociales

Vol.17,\#12, Diciembre 2009

http://revista-redes. rediris.es

Las redes completas y las redes personales son dos tipos de objetos diferentes construidos por dos métodos de observación diferente. Pero ambos comparten orientaciones teóricas comunes relativas a los modelos de racionalidad de los actores y de las limitaciones y oportunidades que los sistemas de relaciones implican, utilizan ciertas técnicas similares para identificar a los alteri y utilizan algunos indicadores sociométricos análogos. Los dos tipos de redes pertenecen a niveles diferentes: las redes personales siempre son subconjuntos de las redes englobantes, tanto si se trata de redes cerradas o abiertas, del mismo modo que una familia nuclear es un subconjunto de una red de parentesco. A nivel de una red completa el análisis a) se centra en un caso, b) muestra una organización de las relaciones que pueden escapar a la conciencia de los actores, c) permite la observación sistemática de los subgrupos, de sus interconexiones, de posiciones poco frecuentes ocupadas por actores centrales o intermediarios. A nivel de las redes personales, el análisis a) identifica regularidades, b) se limita a lo que perciben los actores, c) permite a menudo un análisis más sistemático de las pertenencias múltiples de los actores y de la variedad de los roles que adoptan en distintas relaciones, d) si se aplica a partir de una muestra representativa, permite hacer generalizaciones sobre la sociedad de referencia.

B) Orientaciones teóricas más que una teoría unificada

¿Podemos decir que las redes sociales sean objeto de una teoría unificada? John Barnes nos daba una respuesta negativa: él pensaba que no hay nada que se parezca a una teoría de las redes sociales y que tal vez no la hubiese nunca. Adoptaba una posición prudente. Diez años después, Ronald Burt escribía “el análisis de redes no es un corpus unificado de conocimientos acumulativos a lo largo de los años... es una federación laxa de aproximaciones que progresan en varios frentes gracias a los esfuerzos teóricos de muchas personas"3 (1980, p. 79). Poco después de dicha afirmación, a partir de los años 80 los investigadores trabajando con análisis de redes sociales hacen un esfuerzo por sentar los principios teóricos compartidos subyacentes de lo que Ilaman análisis estructural. El análisis estructural considera la estructura social como una red de relaciones entre actores sociales, como una red de redes interdependientes. Barry Wellman, fundador de la INSNA, afirma en 1988 que se puede identificar un núcleo de cinco ideas comunes al los analistas de redes sociales: 1. Las estructuras de relaciones tienen un poder explicativo más importante que los atributos personales de los

\footnotetext{
${ }^{3}$ « Network analysis is not a single corpus of knowledge cumulating with each passing year. [...] A loose fédération of approaches, progressing on many fronts as a result of the efforts of many persons, is currently refferenced as network analysis. »
} 
REDES- Revista hispana para el análisis de redes sociales

Vol.17,\#12, Diciembre 2009

http://revista-redes. rediris.es

miembros que componen el sistema. 2. Las normas emergen de la interacción y en función de la localización en la estructura de relaciones existentes. 3. Las estructuras sociales determinan el funcionamiento de las relaciones diádicas. 4. El mundo está formado por redes y no por grupos. 5. Los métodos estructurales complementan y suplantan los métodos individualistas.

Unos años después Degenne y Forsé (1994) sitúan el análisis estructural (o según la expresión de su cuño: interaccionismo estructural) de forma teóricamente más precisa entre las tradiciones holistas e individualistas. Como las corrientes holistas (p.e teorías Durkheimianas, Marxistas o Bourdieusianas) el análisis estructural presupone que los actores tienen márgenes de acción limitados por la estructura social, pero de modo más débil, no se trata de una determinación fuerte de los actos. Comparte también la idea de que la estructura no se reduce a una suma de acciones individuales (aunque estas inciden en ella). La estructura ejerce un condicionamiento formal que deja al individuo libre de sus actos aunque, teniendo en cuenta este condicionamiento, todo no le está permitido. También supone como el individualismo metodológico (p.e. siguiendo una tradición más Weberiana de la sociología o posiciones como las de Boudon o Coleman) que los actores tienen motivaciones e intereses y actúan de forma racional para satisfacerlos. Pero también tiene en cuenta, como la perspectiva interaccionista (al estilo de los autores de la Escuela de Chicago, Mead, Blumer, Goffmann etc), el hecho de que los actores no están aislados, sino que son interdependientes y ajustan sus relaciones mediante la interacción.

La existencia de las relaciones obliga a formular los intereses como relativos y sometidos a las limitaciones y oportunidades ofrecidos por la estructura en que los individuos están inmersos. Al mismo tiempo que los actores están condicionados por la estructura social concreta en que se hallan, la (re)producen por medio de sus interacciones cotidianas. Así pues aparece un bucle entre la acción y las relaciones existentes, dado que la acción puede consistir a crear una relación, modificando con ello la red, que a continuación tendrá una influencia en la acción y así sucesivamente (Burt, 1982). Se trata pues de una concepción teórica dinámica. 


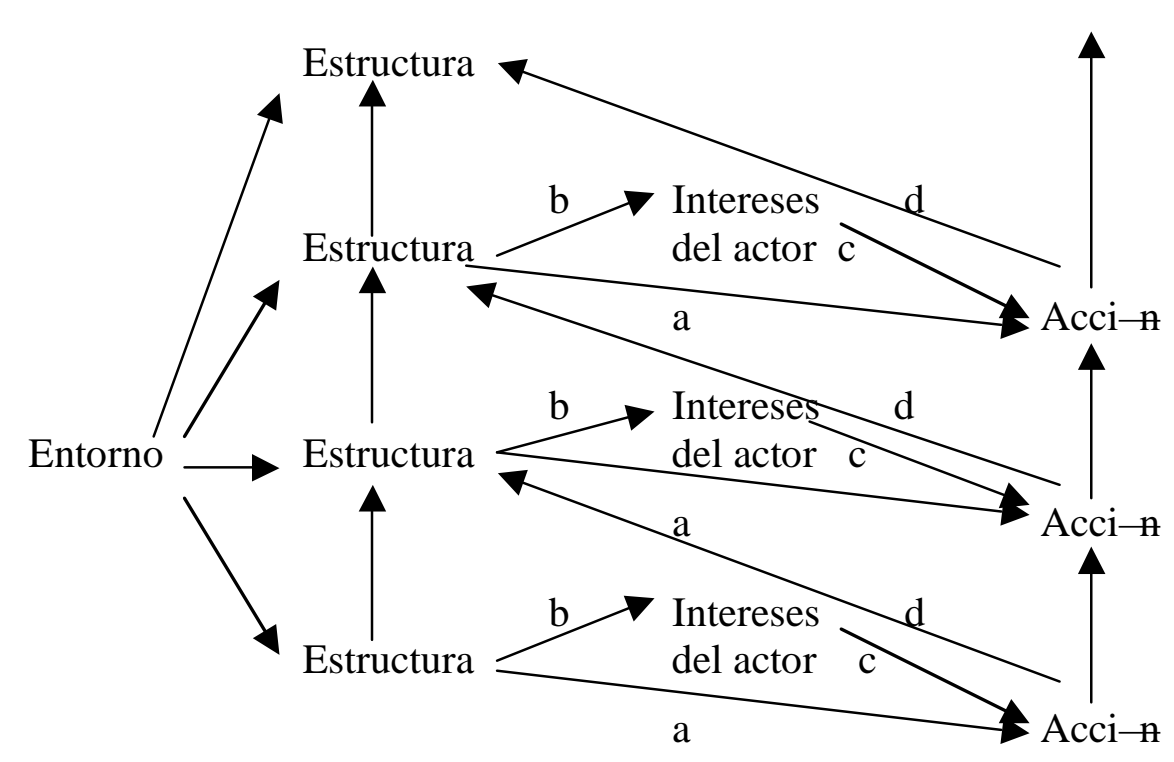

Macro

Meso

Micro

Tiempo

Figura 1. Elementos dinámicos del interaccionismo estructural.

Los actores de la red son particularizados y limitados o favorecidos por sus relaciones recíprocas, pero no determinados: disponen de márgenes de libertad variados que dependen de la organización de la red y de las posiciones que ocupan en ella y que utilizan en función de sus motivaciones para llevar a cabo acciones, que a su vez dejarán una huella en la estructura. Una cuestión crucial para la acción es la posición del actor en la estructura, que nunca está afianzada definitivamente.

Más tarde, Michel Forsé y Simon Langlois insistirán en que se pueden identificar al menos actitudes epistemológicas comunes a los especialistas del análisis de redes que "agrupa a través del mundo los investigadores de un campo quienes, aún sin compartir exactamente las mismas perspectivas teóricas, tienen en común una concepción de la sociología como ciencia positiva y acumulativa" (1997, p. 28).

La diversidad aparente no puede, en definitiva ocultar un importante fondo de ideas comunes. Para empezar la idea de que una red puede tener regularidades, una cierta organización de las relaciones. Se llama entonces estructura (en un primer sentido débil de la palabra estructura) a las principales características empíricas, medidas por índices, construidas por el examen de las matrices que registran las relaciones entre actores: densidad, conectividad, segmentación en cliques o clases de equivalencia estructural, distancias, centralidad, etc. Después, ¿cómo explicar dichas regularidades? Se puede suponer de manera teórica que el establecimiento 
REDES- Revista hispana para el análisis de redes sociales

Vol.17,\#12, Diciembre 2009

http://revista-redes. rediris.es

de una relación o la imposibilidad de crearla dependen de la manera en que las relaciones existentes ya están organizadas. Entonces diremos que la red constituye un sistema relacional, concepto más teórico que el de red, dado que definir un sistema supone que se formulen hipótesis sobre la interdependencia de las relaciones entre ellas. Así pues, podemos llamar estructura a las principales reglas que aseguran la producción de dicho sistema particular (Ferrand, 1997). De esta manera empleamos la palabra estructura en su sentido fuerte para designar un modelo generativo (el tabú del incesto es una de las reglas estructurales del parentesco, pero es necesario definir algunas más para poder definir un sistema de parentesco particular).

\section{I mplicaciones de la teoría en el método}

\section{A) Antes de la perspectiva de redes, la aproximación atributista}

Antes de la aparición del análisis de redes sociales, tal vez la aproximación metodológica más utilizada en sociología fuese el llamado "atributismo", como lo dan en llamar Lozares, Lopez y Saus (2007). En esta aproximación se enumeran los actores sociales (individuos o actores colectivos) objeto de estudio que son caracterizados según sus atributos ordenados en distintas variables (sexo, edad, tendencia política etc.). Los actores son la unidad básica de análisis y sus características organizadas en variables son sometidas a análisis estadísticos y algebraicos, sirviendo para clasificar a los actores e interpretar sus acciones. Esta tradición metodológica comienza con los estudios de Durkheim sobre el suicidio y es ampliamente fomentada por Lazarsfeld, quien crea el modelo de la encuesta representativa cuantitativa por cuestionario, posteriormente en los Estados Unidos.

En el modelo "atributivo" los actores son por lo tanto clasificados a priori según sus características. También, al utilizarse métodos de análisis estadísticos, es obligado considerar en la extracción y el tratamiento de los datos que los actores observados son independientes los unos de los otros.

La interpretación de los resultados de los diferentes análisis se hará en función de las orientaciones teóricas subyacentes a la investigación. Por ejemplo, si la aproximación es de tipo holista, se podrá interpretar el comportamiento del actor como el resultado de las determinaciones dadas por sus atributos, que son una expresión de la estructura social: por el hecho de ser mujer, obrero, joven, de religión católica... la persona se verá condicionada a actuar de tal o cual manera. Una interpretación funcionalista deducirá que el comportamiento deriva de las normas que se aplican en tales contextos. Una interpretación derivada del individualismo-metodológico supondrá que, dadas las posiciones de partida, el actor 
REDES- Revista hispana para el análisis de redes sociales

Vol.17,\#12, Diciembre 2009

http://revista-redes. rediris.es

habrá considerado más interesante y racional comportarse así para maximizar sus probabilidades de éxito. En cualquier caso, en todas estas aproximaciones, los atributos son la base de la clasificación de los actores y de la interpretación de sus actos.

$\begin{array}{cc}\text { Mujer } & \text { Mujer } \\ \text { Joven } & \text { Joven } \\ \text { Atea } & \text { Atea }\end{array}$

$\begin{array}{cc}\text { Hombre } & \text { Hombre } \\ \text { Mayor } & \text { Mayor } \\ \text { Católico } & \text { Católico }\end{array}$

Acción

Acción

Figura 2. El modelo de actor social del atributismo.

La base de datos típica de la aproximación atributista es una matriz rectangular de dos modos (la naturaleza de los objetos en línea y en columna es diferente). Por convención se introducen los actores sociales observados en línea y sus atributos organizados en variables en columna.

\begin{tabular}{|l|l|r|l|l|l|}
\hline $\begin{array}{c}\text { Actores sociales } \\
\text { observados }\end{array}$ & $\begin{array}{c}\text { Variable 1 } \\
\text { (sexo) }\end{array}$ & $\begin{array}{c}\text { Variable 2 } \\
\text { (edad) }\end{array}$ & $\begin{array}{l}\text { Variable 3 } \\
\text { (religión) }\end{array}$ & ... & Variable M \\
\hline Actor 1 & Hombre & 27 & Católico & & A \\
\hline Actor 2 & Mujer & 48 & Ateo & & A \\
\hline Actor 3 & Hombre & & & & B \\
\hline$\ldots$ & & 62 & Católica & & \\
\hline Actir N & Mujer & & C \\
\hline
\end{tabular}

Tabla 1. Base de datos típica del atributismo. 
REDES- Revista hispana para el análisis de redes sociales

Vol.17,\#12, Diciembre 2009

http://revista-redes. rediris.es

\section{B) La aproximación metodológica interaccionista estructural}

La aproximación metodológica del interaccionismo estructural difiere necesariamente de la atributiva por el estatus teórico que se confiere a las relaciones entre los actores sociales y por la naturaleza de los datos con que, por lo tanto, se trata. En lo que se refiere a la posición teórica, Wellman (1988) critica las explicaciones atributistas arguyendo que la característica distintiva de la sociología es la idea de que los modelos de relaciones sociales entre las partes elementales de los sistemas sociales condicionan el comportamiento individual. Según él, esta idea es la que distingue la sociología de la psicología o la economía que se basan en postulados individualistas.

Sin descartar completamente el interés de conocer los atributos de los actores, la focalización interpretativa y analítica en los modelos de relaciones entre los actores sociales, tiene consecuencias evidentes en el método. Primero, es importante averiguar cuáles son las relaciones entre los actores, lo que exige técnicas de investigación y recogida de datos específicas (los llamados generadores de nombres ${ }^{4}$ ). Segundo, el examen de las relaciones y de la interdependencia de los actores sociales invalida el uso de la estadística (que postula la independencia de las observaciones). Los métodos de análisis tratan directamente la naturaleza relacional de la estructura social, lo que exige la creación de modelos de análisis específicos a partir de la teoría de grafos y del cálculo matricial ${ }^{5}$. Tercero, la clasificación de los actores en función de sus modelos de relación no puede ser realizada sino a posteriori, en función de los tipos de relaciones sociales compartidas, una vez la investigación y los análisis han sido realizados.

\footnotetext{
${ }^{4}$ Los generadores de nombres son preguntas que invitan a los encuestados a citar aquellas personas con quienes están vinculados con un cierto tipo de relación. A continuación se suelen detallar las características de las relaciones así como de las personas implicadas. Para una presentación más detallada de los generadores de nombres se pueden ver los trabajos de Campbell y Lee (1991), Requena Santos (1996) o Ferrand y de Federico (2005)

${ }^{5}$ Para la implementación actual de los avances en los modelos de análisis véanse los programas UCINET, Pajek y StOCNET, para su uso véase Molina, Quiroga, Martí, Maya Jariego y de Federico (2006).
} 
REDES- Revista hispana para el análisis de redes sociales Vol.17,\#12, Diciembre 2009

http: // revista-redes. rediris.es

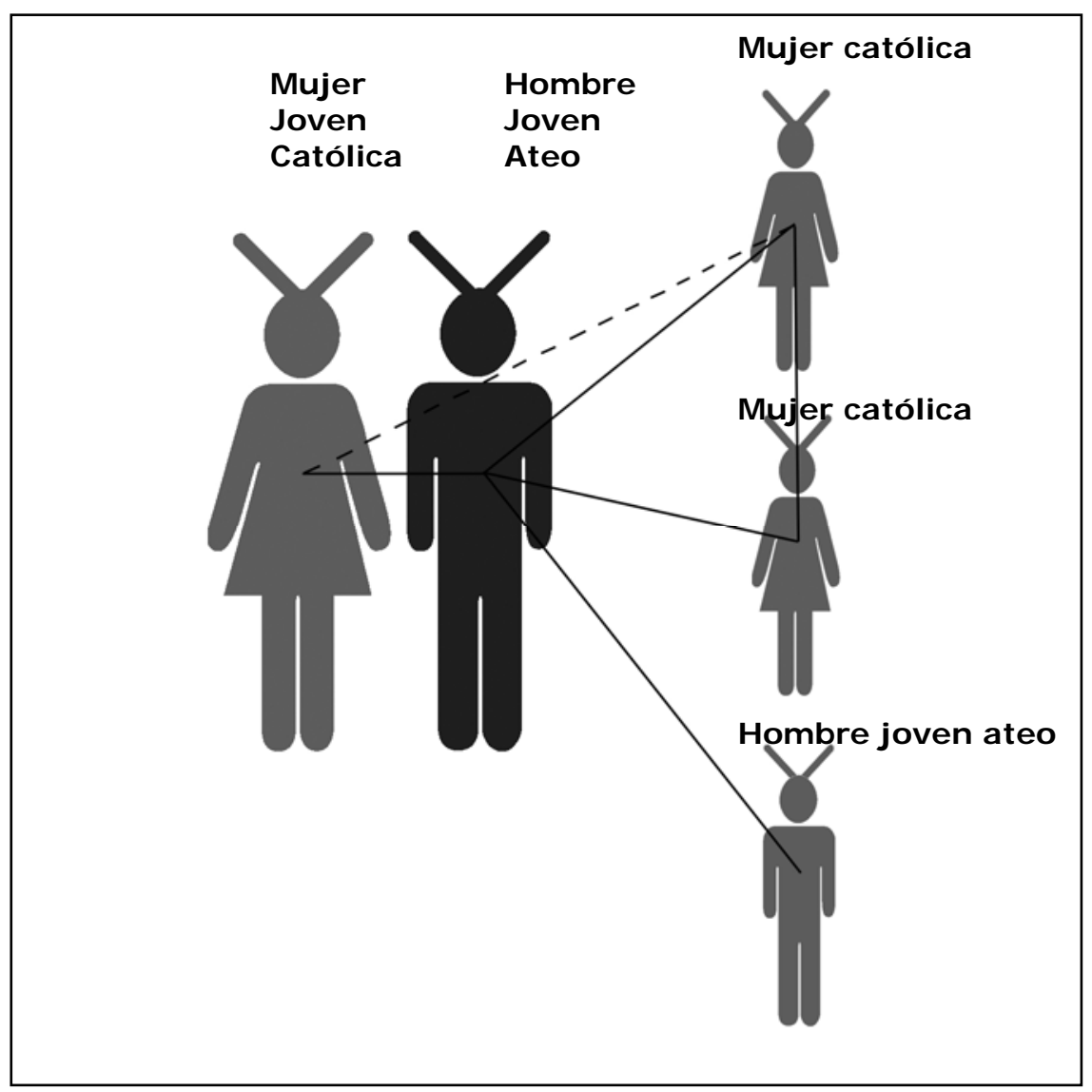

Figura 3. El modelo de actor social del interaccionismo estructural.

El comportamiento de los actores se interpreta a partir de las limitaciones y oportunidades que la estructura de relaciones impone a la acción y no sólo en términos de valores internalizados por los actores. Las relaciones típicas entre más de un actor afectan conjuntamente el comportamiento de cada miembro de la red. Se presta una especial atención a la posición de los actores y la posición de las relaciones. Los actores interactúan, negocian, se ajustan, se ponen de acuerdo, se hacen presión... eso tiene un efecto en las normas emergentes, sobre las acciones de los miembros del sistema $y$, a término, afecta la transformación de la estructura.

\section{C) Una ilustración a propósito del SIDA}

Un ejemplo de las diferencias de interpretación entre la aproximación atributiva y la interaccionista estructural se puede extraer de las investigaciones relativas al SIDA llevadas a cabo en Francia a finales de los ochenta y principios de los noventa. En un contexto en que los mecanismos de transmisión del SIDA ya eran conocidos, se trataba de orientar las campañas de prevención con un conocimiento de las prácticas sociales preciso para que fuesen más eficaces. Para ello la Agencia Nacional de Investigación sobre el Sida planteó una gran investigación 
REDES- Revista hispana para el análisis de redes sociales

Vol.17,\#12, Diciembre 2009

http://revista-redes. rediris.es

interdisciplinar sobre los comportamientos sexuales de los franceses en que apeló a sociólogos, antropólogos, psicólogos, psicoanalistas, epidemiólogos etc. En esta encuesta se utilizaron y pusieron a prueba diferentes aproximaciones teóricas.

Una aproximación holista estructural-funcionalista podría interpretar que, en función de la posición social, los actores están condicionados en sus aprendizajes, normas y comportamientos y se conducirán de tal o cual manera, de forma casi automática, según su socialización. Así pues, se puede esperar tal o cual comportamiento según se sea hombre, mujer, más joven o menos, según las orientaciones religiosas con que se creció etc. (véase la figura 2). Este tipo de aproximación puede parecer relativamente pesimista respecto a una campaña de prevención de choque a corto plazo, pero puede concebir efectos a largo plazo en el marco de la transformación de los valores y la inclusión de la información en las agencias de socialización (p.e la escuela).

Una aproximación afiliada al individualismo metodológico entenderá que los actores son racionales y, dada la información que reciben sobre los riesgos de transmisión, su capacidad para asimilarla (socialmente diferenciada) y sus accesos a diferentes recursos (socialmente diferenciados) adoptarán las posiciones más interesantes y racionales. Con una argumentación diferente, motivados y racionales, pero compartiendo las ventajas y desventajas de otros individuos con los mismos atributos, en esta aproximación también se entenderá que el comportamiento será similar para personas con las mismas características. Este tipo de aproximación parece mucho más optimista respecto a la funcionalidad de los mensajes de prevención a corto plazo. Dentro de esta aproximación se puede entender que una buena campaña informativa que llegue a todos los públicos debería ser suficiente para que los individuos adopten comportamientos racionales sin riesgos. Un ejemplo de mensaje podría ser: "en una nueva relación sexual, ponte un preservativo".

Ambas aproximaciones desestiman sin embargo que, en el caso concreto de los riesgos de transmisión del sida por vía sexual, los comportamientos sexuales en principio no son solo cosa de un individuo socializado o racional, sino que forman parte de una relación entre dos personas que frecuentemente incluye otros contenidos aparte de las actividades sexuales. Los actos sexuales compartidos frecuentemente son objeto de intercambios de palabras entre los implicados, de negociaciones y ajustes. 
REDES- Revista hispana para el análisis de redes sociales

Vol.17,\#12, Diciembre 2009

http://revista-redes. rediris.es

De hecho, no sólo los actos sexuales en los que se transmite el sida son cosa de dos actores vinculados por una relación, dicha relación además suele estar inscrita en un sistema de relaciones en que circulan normas, representaciones, juicios, sobre lo que está bien o no hacer. Según como sea el clima normativo del entorno del actor y los riesgos relativos a la reputación, éste puede verse influido de tal o cual manera. Tanto mas si se trata de las personas que son sus confidentes directos en temas de amores y sexualidad.

La aproximación interaccionista estructural es también optimista respecto a la funcionalidad de las campañas de prevención, siempre y cuando los mensajes y consejos sean discutidos y formen parte de los intercambios de palabra, de la interacción, del ajuste y el control recíproco dentro de las relaciones sexuales o triádico en aquellas relaciones en que se habla de sexo. Así pues, algunos mensajes de prevención desde el interaccionismo estructural podrían ser "habla con tu pareja sobre el sida y sus riesgos", "si lo conoces desde hace poco, dile a tu pareja que se ponga un preservativo", pero también "habla con tus amigos del sida y sus riesgos" y "dile a tus amigos que utilicen preservativos".

\section{Niveles y modelos de análisis típicos del interaccionismo estructural}

\section{A) Los niveles de análisis}

En la aproximación interaccionista estructural aparecen diferentes niveles de análisis. El primer nivel es el de los actores de la red y su composición en términos de atributos. En este nivel los análisis son similares a la aproximación atributiva y las bases de datos utilizadas son idénticas a las de dicha perspectiva.

El segundo nivel es el de las relaciones típicas existentes en la red. En este punto sólo consideramos las díadas compuestas por tres elementos, los dos actores ligados A y B (al encuestado se le llamará ego y a cada relación que mencione alter1, alter2, alter3, etc.) y la relación específica que los vincula cuya forma (nivel de implicación, antigüedad, frecuencia de interacción, polivalencia etc.) y contenido (contenidos de interacción típicos entre los actores) serán especificados. Las bases de datos para analizar las relaciones son parecidas a las de la aproximación atributiva con la diferencia de que las observaciones no son los actores sino las relaciones entre los encuestados y las diferentes personas con quienes están vinculados. 
REDES- Revista hispana para el análisis de redes sociales

Vol.17,\#12, Diciembre 2009

http:// revista-redes. rediris.es

\begin{tabular}{|l|l|r|l|r|l|l|}
\hline $\begin{array}{c}\text { Actores sociales } \\
\text { observados }\end{array}$ & $\begin{array}{c}\text { Variable 1 } \\
\text { ego (sexo) }\end{array}$ & $\begin{array}{c}\text { Variable 2 } \\
\text { ego (edad) }\end{array}$ & $\begin{array}{c}\text { Variable 1 } \\
\text { alter (sexo) }\end{array}$ & $\begin{array}{c}\text { Variable 2 } \\
\text { alter } \\
\text { (edad) }\end{array}$ & $\begin{array}{c}\text { Variable 1 } \\
\text { relación } \\
\text { (rol) }\end{array}$ & $\begin{array}{c}\text { Variable 2 } \\
\text { relación } \\
\text { (consejo) }\end{array}$ \\
\hline Ego 1 & Hombre & 31 & Hombre & 32 & Amigos & No \\
\hline Ego 1 & Hombre & 31 & Mujer & 27 & Pareja & Si \\
\hline Ego 1 & Hombre & 31 & Mujer & 28 & Confidente & Si \\
\hline Ego 2 & Mujer & 27 & Mujer & 30 & Amigas & Si \\
\hline Ego 3 & Hombre & 48 & Hombre & 54 & Amigos & No \\
\hline Ego 3 & Hombre & 48 & Mujer & 42 & Pareja & Si \\
\hline$\ldots$ & & 62 & Mujer & 65 & Amigas & Si \\
\hline Ego N & Mujer & & & \\
\hline
\end{tabular}

Tabla 2. Bases de datos para análisis relacional.

El tercer nivel de análisis es el propiamente reticular referente a las redes sociales que presentan las interdependencias de las relaciones. La forma dependerá del método por el que se haya optado, describiendo redes personales, o completas. En cualquiera de ambos casos existirá una dimensión triádica del análisis, es decir, que se examinará la interdependencia de las relaciones entre ellas. Se tratará típicamente de matrices cuadradas de un solo modo, dado que encontramos el mismo tipo de objetos en línea y en columna (los actores) y en el centro de la matriz encontramos la presencia / ausencia de relaciones, a veces asociado al tipo o calidad de la relación.

\begin{tabular}{|c|c|c|c|c|c|c|}
\hline & Ego 1 & Ego 2 & Ego 3 & . . & Ego N & \multirow{4}{*}{$\begin{array}{l}0 \text { No hay relación } \\
1 \text { Amigos }\end{array}$} \\
\hline Ego 1 & & 0 & 1 & $\ldots$ & 1 & \\
\hline Ego 2 & $\underline{0}$ & & 2 & $\ldots$ & 2 & \\
\hline Ego 3 & 1 & 2 & & $\ldots$ & 1 & \\
\hline & & & & & & 2 Pareja \\
\hline Ego N & 2 & 2 & 1 & & & \\
\hline
\end{tabular}

Tabla 3. Bases de datos en el análisis de redes sociales

\section{B) Los modelos de análisis}

La red como forma colectiva toma, según los análisis, bien el estatus de variable independiente que explica las estrategias de los actores o de los efectos colectivos, bien el estatus de variable dependiente, explicada por los motivos y principios que conducen a los actores a crear, mantener y suprimir relaciones. Encontramos en el análisis de redes sociales cuatro tipos principales de modelos de análisis:

- El primero trata de comprender las conductas de los actores a partir de las relaciones en que están implicados y de las posiciones particulares que ocupan en una red. Se considera que las posiciones en dichas estructuras son principios 
REDES- Revista hispana para el análisis de redes sociales

Vol.17,\#12, Diciembre 2009

http://revista-redes. rediris.es

explicativos tan poderosos o más que las características personales que los definen fuera de todo contexto: edad, ingresos, nivel educativo, etc. (Wellman y Berkowitz 1988 , p. 30). Se tienen en cuenta tres efectos. Un efecto cognitivo y normativo : mostramos que la red puede influir en las orientaciones, las opiniones, las normas y las creencias de los actores (Ferrand y Mounier, 1996). Un efecto instrumental: las relaciones ofrecen posibilidades de movilización de recursos de todo tipo, por lo tanto medios de acción (Lin, 2001). Finalmente un efecto de control: las relaciones imponen límites a las acciones posibles y controlan la acción.

- El segundo trata de explicar los efectos colectivos a partir de las características de la red en cuestión. El tipo de estructura condiciona la difusión de la información, el control social y la cohesión, la diferenciación del poder y de los conflictos, etc. (Galaskiewicz, 1985 ; Lazega, 2001).

- El tercer tipo de análisis tiene como objetivo explicar la red misma, las razones y las condiciones de su existencia, con sus propiedades particulares y sus condiciones de transformación. Actualmente se están logrando progresos importantes tanto para definir los modelos estadísticos que permiten evaluar las transformaciones de una red completa en momentos diferentes como de los modelos de simulación de dichas transformaciones (Snijders, 2005 ; de Federico de la Rúa, 2005 ; Maya Jariego y Molina, 2008).

- El cuarto tipo de análisis, finalmente, tiene por objetivo explicar las características de las redes personales (lo que autoriza a una interpretación parcial de una red global) a partir de la influencia de modelos culturales (Heran, 1988 ; Fischer, 1982), por una intención estratégica de los actores o por la influencia de constricciones estructurales. En este sentido, una relación diádica entre dos actores puede ser entendida en función de su imbricación (embeddedness) entre otras relaciones del actor.

Para terminar, definir un fenómeno o una entidad en términos de red de relaciones es casi siempre posible y razonable: pocas realidades sociales no comportan relaciones entre actores dotadas de una mínima organización, por lo tanto descubrir la existencia de una red es afirmar lo evidente. La cuestión interesante es mostrar cuáles son las particularidades estructurales de la red que forma dicha realidad, tarea totalmente diferente y apasionante. Sólo es posible producir proposiciones científicamente interesantes al realizar comparaciones entre las propiedades estructurales y los efectos de tipos diferentes de redes.

\section{Bibliografía}


REDES- Revista hispana para el análisis de redes sociales

Vol.17,\#12, Diciembre 2009

http://revista-redes. rediris.es

Barnes John A., (1954). Class and comittees in a Norwegian Island parish, Human Relations, $n^{\circ} 7$, p. 39-58.

Burt Ronald S., (1980). Models of network structure, Annual Review of Sociology, $\mathrm{n}^{\circ}$ 6, p. 79-141.

Burt Ronald S., (1982). Toward a structural theory of action. - New York, Academic Press, $381 \mathrm{p}$.

Campbell Karen E. y Lee Barrett A., (1991). Name generators in surveys of personal networks, Social Networks, vol. 13, n³, p. 203-221.

Degenne Alain y Forsé Michel (1994). Les réseaux sociaux. - Paris, Armand Colin, 288 p. (Trad. Anglaise : Introducing social networks, London, SAGE, 248 p.).

Federico de la Rúa (de) Ainhoa, (2005). El análisis dinámico de redes sociales con SIENA. Método, discusión y aplicación, Empiria Revista de metodología de ciencias sociales, $n^{\circ} 10$, p. 151-184.

Ferrand Alexis (1997). La structure des systèmes de relations, L'Année sociologique, vol. 47, $n^{\circ} 1$, p. 31-54.

Ferrand Alexis y Mounier Lise (1996). Talking about sexuality : An analysis of relations between confidants, en Michel Bozon y Henri Leridon (éd.), Sexuality and the social sciences, p. 265-288. - Aldershot, Dartmouth, 357 p.

Ferrand Alexis y de Federico Ainhoa (2005). Methods of social network analysis, in : Graziella Casselli, Jacques Vallin y Guillaume Wunch (éd.), Demography: Análisis and Síntesis. A treatise in population. Volume IV, Section II Observation auxiliary methods, teaching and research. p. 745-781. - Elsevier, Academia Pres.

Fischer Claude S. (1982). To dwell among friends : personal network in town and city. - Chicago, University Press, 451 p.

Forsé Michel y Langlois Simon (1995). Analyse structurelle comparée du changement social. Le cas de la France et du Québec, in : Michel Forsé y Simon Langlois (dir.), Tendances comparées des sociétés post-industrielles, p. 215-261. Paris, PUF, $270 \mathrm{p}$.

Freeman Linton C. (2004). The development of social network analysis. A study in the sociologie of science. - Vancouver, Empirical press, 205 p.

Galaskiewicz Joseph (1985). Social organization of the urban grant economy : a study of business philanthropy and non profit organizations. - New York, Academic Press.

Héran François (1988). La sociabilité, une pratique culturelle, Economie et Statistiques, $n^{\circ} 216$, p. 3-22.

Lazega Emmanuel (2001). The collegial phenomenon : the social mechanisms of cooperation among peers. - Oxford, Oxford University Press, 346 p. 
REDES- Revista hispana para el análisis de redes sociales

Vol.17,\#12, Diciembre 2009

http://revista-redes. rediris.es

Lin Nan, 2001. - Social capital: a theory of social structure and action. Cambridge, Cambridge University Press, 278 p.

Lozares Colina Carlos, López Roldán Pedro, Saus Moreno, Judith (2007). Del paradigma atributivo al relacionista y viceversa, $V$ Mesa Hispana para el análisis de redes sociales. XXVII International Sunbelt Social Network Conference - Corfú, Grecia, 2 de mayo de 2007.

Maya Jariego Isidro y Molina J osé Luis (2008). Monográfico análisis dinámico de las redes personales REDES. Revista hispana para el análisis de redes sociales. Volumen 15.

Molina José Luis, Quiroga Agueda, Martí Joel, Maya Jariego Isidro, de Federico Ainhoa (2001). Talleres de autoformación con programas informáticos de análisis de redes socials. - Barcelona, Universitat Autònoma de Barcelona, 107 p.

Moreno Jacob L. (1934). Who shall survive? - Washington (DC), Nervous and Mental Disease Publishing Company.

Requena Santos Félix (1996). Redes sociales y cuestionarios. - Madrid, Cuadernos Metodológios. Centro de investigaciones sociológicas \& Siglo XXI, 57 p.

Scott John, 1992. - Social network analysis: a handbook. - Newbury Park (Californie), Sage, $208 \mathrm{p}$.

Snijders, Tom (2005). Models for Longitudinal Network Data, in : Peter Carrington, John Scott, \& Stanley Wasserman (eds.), Models and methods in social network analysis. p.123-151 - New York: Cambridge University Press.

Wellman Barry y Berkowitz Stephen D. (ed.) (1988). Social structures. A network approach. - Cambridge, Cambridge University Press, 508 p. 